\title{
POLARIMETRIC FEATURE EVALUATION FOR STRICKEN MAN-MADE OBJECT DETECTION USING FDTD POLARIMETRIC SCATTERING ANALYSIS
}

\author{
Ryoichi Sato ${ }^{1 *}$, Yoshio Yamaguchi ${ }^{2}$, Hiroyoshi Yamada ${ }^{2}$, Sang-Eun Park ${ }^{2}$ \\ ${ }^{1}$ Faculty of Education, Niigata University \\ 8050, 2-no-cho, Ikarashi, Nishi-ku, Niigata, 950-2181 Japan \\ e-mail: sator@ed.niigata-u.ac.jp \\ ${ }^{2}$ Department of Information Engineering, Niigata University, Japan
}

\begin{abstract}
This paper investigates polarimetric scattering characteristics for accurately detecting stricken man-made objects. In order to understand the polarimetric scattering features generated from various damaged man-made buildings, we carry out the Finite-Difference Time-Domain (FDTD) polarimetric scattering analysis for deformed and/or collapsed man-made target models. The dependency of the polarimetric scattering feature on the variation of squint angle is in detail investigated. It is confirmed from the estimation based on various polarimetric analyses that the double-bounce scattering, the co-pol correlation coefficient in LR circular porlarization basis, and its normalized one are useful markers for stricken man-made objects detection/identification.
\end{abstract}

Index Terms - stricken man-made object detection, FDTD analysis, radar polarimetry, polarimetric synthetic aperture radar (PolSAR)

\section{INTRODUCTION}

In the field of disaster monitoring, observation survey method independent of the weather condition and day and night is strongly required under emergent and severe stricken situation. PolSAR may be one of the best solutions for such purpose.

In PolSAR data utilization, man-made object detection/identification is one of the most important subjects. So there are many useful man-made object detection and identification techniques $[1,2,3,4,5,6,7]$. For complex urban area monitoring, however, it is sometimes difficult to distinguish the man-made object from other natural distributed ones, since the polarimetric scattering behavior from the man-made object is strongly dependent on the direction or orientation of their alignment. When the man-made objects are obliquely aligned to the PolSAR's illumination direction,

The authors express their sincere appreciations to JAXA, Japan, for providing valuable ALOS/PALSAR image data sets. This research was partially supported by a Scientific Research Grant-In-Aid (22510188, 25350495) from JSPS (Japan Society for the Promotion of Science), Japan. the detection accuracy of each technique may become low, since the useful polarimetric index for classifying the manmade object (strong double-bounce scattering, large LL-RR correlation coefficient, phase of co-pol ratio, etc. ) may hardly be observed from the oblique object areas.

In this paper, to realize accurate detection for obliquely oriented man-made object even in strcken situation, we shall try to find out an appropriate combination from the existing techniques, by carrying out polarimetric scattering analysis for simplified man-made objects. Here, we utilize the FiniteDifference Time-Domain (FDTD) method for the analysis. We here consider not only well-regulated man-made object model but also deformed and/or collapsed ones, with assumption that the residential houses are stricken by terrible disaster as big earthquake.

\section{FDTD POLARIMETRIC SCATTERING ANALYSIS}

As depicted in Fig.1, we carry out polarimetric scattering analysis for simple model, which consists of quad dielectric rectangular parallelepipeds on finite circular plate with rough surface. The rectangular parallelepipeds are considered as a simplified man-made buildings. We consider five models as follows. Fig.1 (a) is well-regulated model (each parallelepiped is well-regulated). Figs.1 (b) and (c) are deformed models (the parallelepipeds are inclined to left or backward to radar illumination direction). Also, Figs.1 (d) and (e) are collapsed models (one of the parallelepipeds is collapsed or removed). The FDTD method [8] is here utilized to obtain the elements $\left(S_{H H}, S_{V V}, S_{H V}\right)$ of the scattering matrix $[S]$. We carry out many existing polarimetric analyses and at least obtain the following indices for accurate detection of man-made object.

- Double-bounce scattering from Model-based scattering power decomposition $[1,2,3]$

- Co-pol. correlation coefficient in LR basis, and the normalized one $[4,5,6]$ 


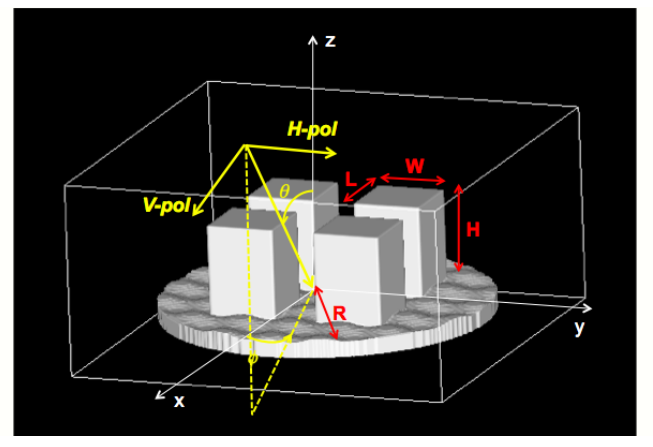

(a) well-regulated model
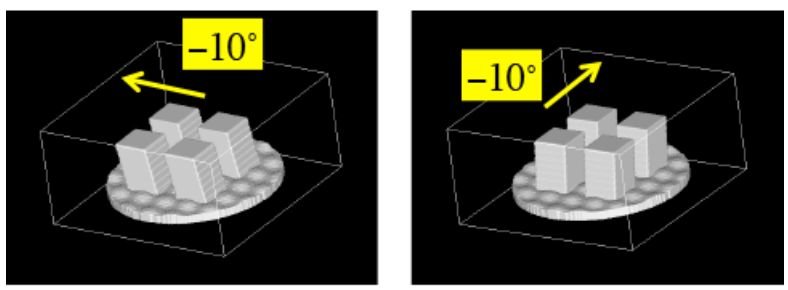

(b) left-deformed

(c) backward-deformed
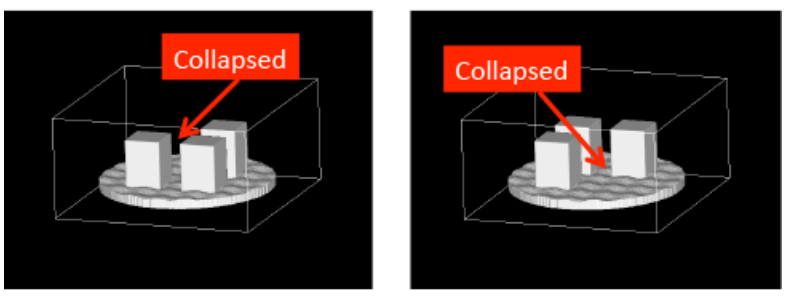

(d) left-back collapsed

(e) right-front collapsed

Fig. 1. Geometry of the problem

We will also execute the other analyses as "Entropy $H$, Angle $\bar{\alpha}$, Anisotropy $A$ " based on Eigen-value decomposition [7], and "Phase of co-pol. ratio in HV basis" in the presentation.

After getting the above indices, we carefully evaluate them and try to find out appropriate combination from them for man-made object detection.

\section{RESULT OF FDTD ANALYSIS}

We shall show the FDTD results for the well-regulated, deformed/inclined, and partially collapsed models, as in Fig.1.

The geometrical parameters of the model are $\mathrm{W}=\mathrm{L}=4.8 \lambda$ (1.2m), $\mathrm{H}=6.4 \lambda(1.6 \mathrm{~m}), \mathrm{D}=1.2 \lambda(0.3 \mathrm{~m})$ and $\mathrm{R}=12.0 \lambda(3.0 \mathrm{~m})$ at L-band frequency $(1.2 \mathrm{GHz})$. The complex relative permittivities are set as $\varepsilon_{r 1}=4.0+i 0.2$ for main parallelepiped part (Concrete) and $\varepsilon_{r 2}=7.0+i 0.1$ for base part of the circular plate (Dry soil) at the same frequency. The base part has si-
Table 1. FDTD fundamental parameters

\begin{tabular}{|l|l|}
\hline Analytical region & $700 \times 700 \times 350$ cells \\
\hline Cubic cell size $\Delta$ & $0.01 \mathrm{~m}$ \\
\hline Time step $\Delta t$ & $1.925 \times 10^{-11} \mathrm{~s}$ \\
\hline Incident pulse & Lowpass Gaussian pulse \\
\hline ABC & PML (8 layer) \\
\hline
\end{tabular}

nusoidal rough surface with $\lambda / 2$ height amplitude. The other fundamental FDTD parameters are shown in Table 1 . The incident or look angle $\theta=\theta_{0}$ is fixed at $44^{\circ}$, and the squint angle $\phi=\phi_{0}$ is variable $\left(1^{\circ}-44^{\circ}\right)$. In order to do statistical evaluation for mean squint angle, the ensemble average processing is carried out for 9 degrees squint angular range.

Figure 2 shows the result of the FDTD analysis obtained by the scattering power decomposition. Here, we utilize the nonnegative eigenvalue decomposition (NNED) [9] and $a$ unitary rotation [10] to the $3 \times 3$ covariance matrix. All figures show the mean squint angle dependency. It is found from the results that the double-bounce scattering (red) is considered as a useful marker of the man-made objects when the squint angle is relatively small (less than $15^{\circ}$ ), regardless of geometry of the stricken building models.

Figure 3 shows the result of the co-pol. correlation coefficient in LR polarization basis $\gamma_{L L-R R}$ [4], and the normalized one $\gamma_{L L-R R}^{\prime}$ [5]. It is observed from Fig.3 (b) that $\gamma_{L L-R R}$ keeps large value for the wide squint angular range (up to about $30^{\circ}$ ). Also, the normalized correlation coefficient $\gamma_{L L-R R}^{\prime}$ shows outstanding large values for relatively large squint angular ranges. Hence, $\gamma_{L L-R R}^{\prime}$ may be one of the candidates as useful polarimetric markers for obliquely oriented stricken man-made object detection.

\section{CONCLUDING REMARKS}

In this paper, we have examined polarimetric scattering characteristics from simplified man-made structure models, in order to improve the accuracy of detection/classification of the stricken man-made object in POLSAR image analysis. Here, the Finite-Difference Time-Domain (FDTD) method has been utilized for the detailed analysis. It has been found from the FDTD analysis that the double-bounce scattering, the co-pol correlation coefficient in LR circular porlarization basis, and its normalized one are very useful tools for finding out peculiar scattering feature for detecting/ classifying deformed and/or partially collapsed man-made objects.

Furthermore, we will investigate the features of other polarimetric indices, by carrying out additional FDTD polarimetric scattering analysis. 


\section{REFERENCES}

[1] A. Freeman and S. L. Durden, "A three-component scattering model for polarimetric sar data," IEEE Trans. Geosi. Remote Sensing, vol. 36, no. 3, pp.963-973, May 1998.

[2] Y. Yamaguchi, T. Moriyama, M. Ishido, and H. Yamada, "Four-component scattering model for polarimetric sar image decomposition," IEEE Trans. Geosi. Remote Sensing, vol. 43, no. 8, pp. 1699-1706, Aug. 2005.

[3] J. van Zyl and Y. Kim, "Synthetic aperture radar polarimetry," Wiley, 2011.

[4] J.-S. Lee and E. Pottier, "Polarimetric radar imaging: from basics to applications," CRC Press, 2009.

[5] D. Schuler, J.-S. Lee, and T. Ainsworth, "Polarimetric sar detection of man-made structures using normalized circular-pol correlation coefficient," Proc. of IGARSS 2006, pp. 1699-1706(CD-ROM), Aug. 2006.

[6] T. Ainsworth, D.L. Schuler, and J.-S. Lee, "Polarimetric sar characterization of man-made structures in urban areas using normalized circular-pol correlation coefficients," Remote Sensing of Environment, vol. 112, pp. 2876-2885, 2008.

[7] S. R. Cloude, "Polarisation: Applications in remote sensing," Oxford Univ. Press, 2009.

[8] A. Taflove and S. C. Hangness, "Computational electrodynamics (3rd ed.)," Artech House, 2005.

[9] J. J. van Zyl, M. Arii, and Y. Kim, "Requirements for model-based polarimetric decompositions," Proc. of the IGARSS 2008, vol. V, pp. 417-420, 2008.

[10] Y. Yamaguchi, A.Sato, W.-M. Boerner, R. Sato, and H. Yamada, "Four-component scattering power decomposition with rotation of coherency matrix," IEEE Trans. Geosi. Remote Sensing, vol. 49, no. 6, pp. 22512258, June 2011.

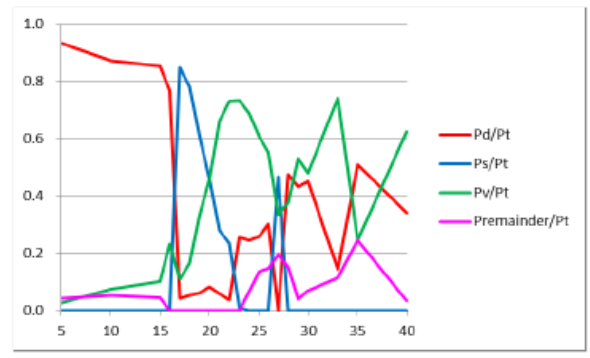

(a) well-regulated

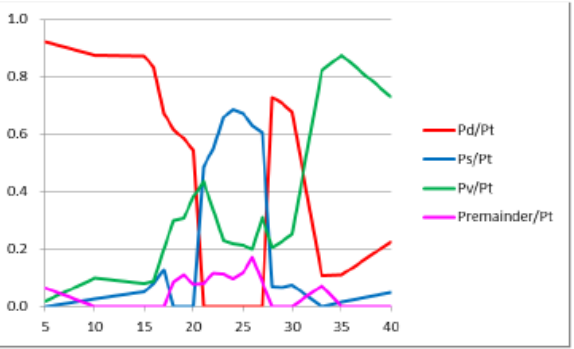

(b) left-deformed

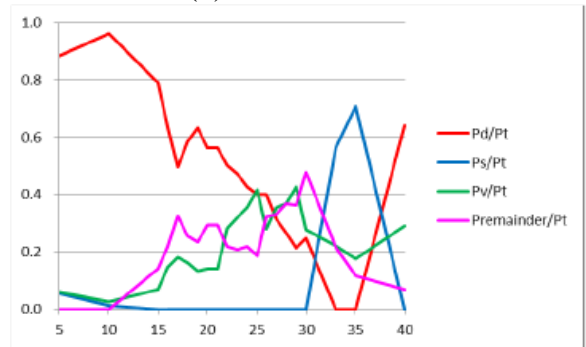

(c) backward-deformed

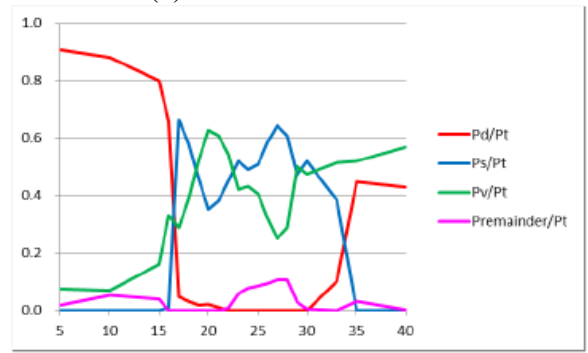

(d) left-back object collapsed

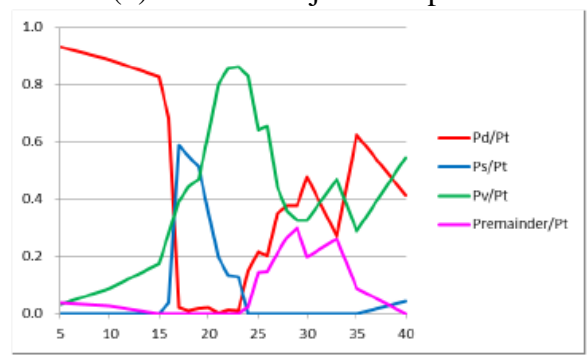

(e) right-front object collapsed

Fig. 2. Result of the FDTD analysis (scattering power decomposition) 


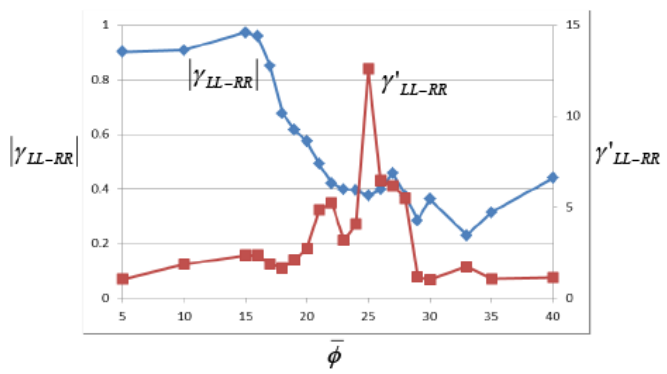

(a) well-regulated

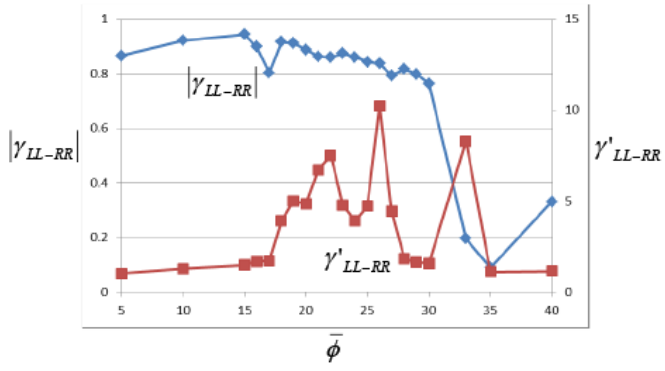

(b) left-deformed

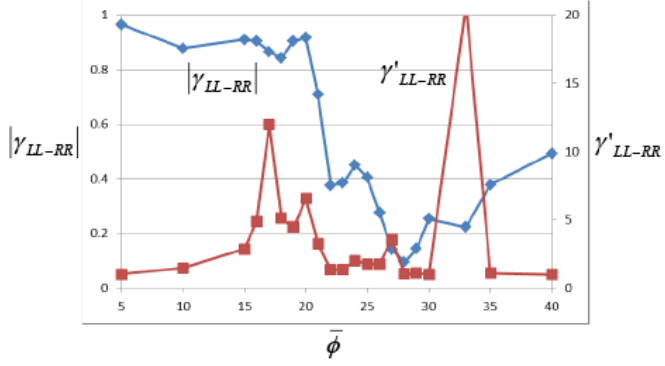

(c) backward-deformed

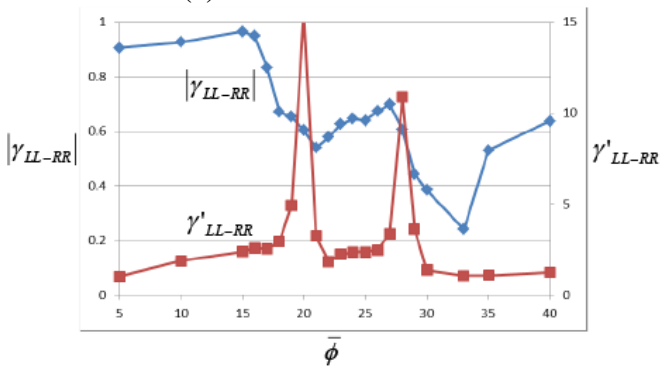

(d) left-back object collapsed

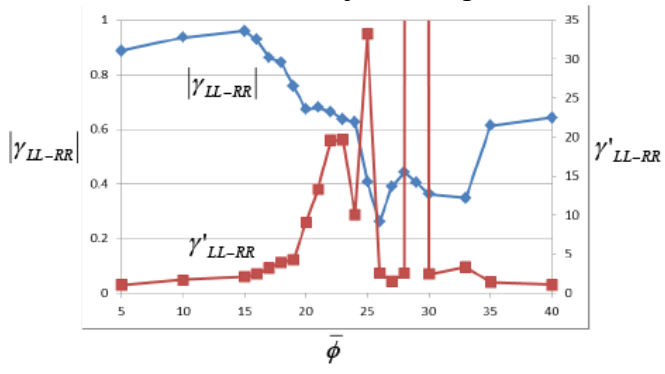

(e) right-front object collapsed

Fig. 3. Result of the FDTD analysis (correlation coefficient in LR circular polarization basis) 